\title{
Specular Path Estimation Errors with ESPRIT, SAGE, and RiMAX in the Presence of Dense Multipath
}

\author{
E. Tanghe ${ }^{1}$, L. Martens ${ }^{1}$, W. Joseph ${ }^{1}$, D. P. Gaillot ${ }^{2}$, M. Liénard ${ }^{2}$, and P. Degauque ${ }^{2}$
}

\begin{abstract}
The estimation accuracy of specular multipath components in radio channels that include dense multipath is investigated. Classical multipath estimation algorithms such as ESPRIT and SAGE do not include dense multipath in their signal model whereas recent ones, such as RiMAX, do. These estimation algorithms are applied to a-priori known synthetic channels which include both specular components (SCs) and dense multipath components (DMC). The estimation errors of the SCs are computed as a function of the DMC power to evaluate the estimator's robustness. The results of this work clearly indicate large estimation errors for the SC parameters when the estimator does not include DMC in its data model.
\end{abstract}

\section{INTRODUCTION}

The signal model of conventional high-resolution multipath estimation algorithms such as ESPRIT [1] and SAGE [2] presupposes that the wireless radio channel consists of a set of discrete propagation paths (specular components or SCs). Additionally, the model also accounts for measurement imperfections by including a noise term that is assumed to be white in both the angular and delay domains. Recent work suggests to also include dense multipath components (DMC) to the signal model of estimation algorithms [3]. DMC is the part of the multipath profile that is continuous in both the angular and delay domains. DMC is modeled as an additive colored noise term and has been included in recently developed estimation algorithms, most notably RiMAX [3].

The physical reality of DMC raises the question how well estimation algorithms which historically do not include DMC into their signal model (ESPRIT, SAGE) estimate the SC part of the channel, and this compared to the performance of a DMC-inclusive estimation algorithm (RiMAX). This question is investigated here.

\section{CHANNEL CONSTRUCTION AND ESTIMATION}

The physical environment chosen for synthesizing channels is a $66 \mathrm{~m} \times 32 \mathrm{~m} \times 10.8 \mathrm{~m}$ sports hall. In this environment, 1000 channels are constructed, where each channel corresponds to randomly chosen positions for the transmitting and receiving antennas. The sampled array response vector $\boldsymbol{h} \in \mathbb{C}^{M_{r} M_{t} M_{f} \times 1}$ (where $M_{r}, M_{t}$, and $M_{f}$ correspond to the numbers of receive antennas, transmit antennas, and frequency points, respectively) can be written as the sum of a deterministic SC part $\boldsymbol{s}$ and a stochastic DMC part $\boldsymbol{d}$. It is assumed that $\boldsymbol{h}$ follows a multivariate circular symmetric complex Gaussian process [3]:

$$
\begin{aligned}
\boldsymbol{h} & =\boldsymbol{s}\left(\boldsymbol{\theta}_{\boldsymbol{S C}}\right)+\boldsymbol{d}\left(\boldsymbol{\theta}_{\boldsymbol{D M C}}\right) \\
\text { and } \boldsymbol{h} & \sim \mathcal{N}_{C}\left(\boldsymbol{s}\left(\boldsymbol{\theta}_{\boldsymbol{S C}}\right), \boldsymbol{R}\left(\boldsymbol{\theta}_{\boldsymbol{D M C}}\right)\right)
\end{aligned}
$$

To construct $\boldsymbol{s}\left(\boldsymbol{\theta}_{\boldsymbol{S C}}\right)$, ray-tracing is used to obtain the 50 strongest specular paths. The sports hall is modeled as a simple box-like structure for the raytracing simulations. Four parameters are associated with each SC (grouped into the parameter vector $\boldsymbol{\theta}_{\boldsymbol{S C}}$ ), namely its Azimuth Of Arrival (AOA), Azimuth Of Departure (AOD), Time delay Of Arrival (TOA), and complex amplitude. On the other hand, $\boldsymbol{d}\left(\boldsymbol{\theta}_{\boldsymbol{D M C} \boldsymbol{C}}\right)$ is fully determined by the channel covariance matrix $\boldsymbol{R}\left(\boldsymbol{\theta}_{\boldsymbol{D M C}}\right)$. In recent models for the $\mathrm{DMC}$, this covariance matrix is assumed to have the following structure involving Kronecker products [3]:

$$
\boldsymbol{R}\left(\boldsymbol{\theta}_{\boldsymbol{D} \boldsymbol{M C}}\right)=\boldsymbol{I}_{\boldsymbol{M}_{\boldsymbol{r}}} \otimes \boldsymbol{I}_{\boldsymbol{M}_{\boldsymbol{t}}} \otimes \boldsymbol{R}_{\boldsymbol{f}}\left(\alpha_{1}, B_{d}, \tau_{d}, \alpha_{0}\right)
$$

, where $I$ represents the identity matrix. In (2), the dense field is modeled as white noise in the angular domains $\left(\boldsymbol{I}_{\boldsymbol{M}_{\boldsymbol{r}}}\right.$ and $\left.\boldsymbol{I}_{\boldsymbol{M}_{\boldsymbol{t}}}\right)$ and as colored noise in the time delay domain $\left(\boldsymbol{R}_{\boldsymbol{f}}\right)$. The DMC power delay profile $\psi(\tau)$ as a function of time delay $\tau$ is typically described by an exponential decay:

$$
\psi(\tau)=\alpha_{1} e^{-B_{d}\left(\tau-\tau_{d}\right)}+\alpha_{0}
$$

, where $\alpha_{1}, B_{d}, \tau_{d}$, and $\alpha_{0}$ are four parameters which fully describe the DMC and are gathered into the DMC parameter vector $\boldsymbol{\theta}_{\boldsymbol{D M C}}$. The DMC parameters were retrieved from channel sounding measurements in the sports hall.

Following the construction of $\boldsymbol{\theta}_{\boldsymbol{S C}}$ and $\boldsymbol{\theta}_{\boldsymbol{D M C}}$, the array response vectors $\boldsymbol{h}$ are calculated according to (1). For this, $4 \times 4$ uniform rectangular antenna arrays were chosen at both receive and transmit side $\left(M_{r}=M_{t}=16\right)$. In addition, a $40 \mathrm{MHz}$ bandwidth centered at $3.5 \mathrm{GHz}$ was considered with a $1 \mathrm{MHz}$ frequency step $\left(M_{f}=41\right)$. Finally, 10 independent observations of $\boldsymbol{h}$ were drawn for each channel. The channel construction process is repeated for three different ratios of the total DMC power $P_{D M C}$ to the total SC power $P_{S C}$, namely $P_{D M C} / P_{S C}$ equal to $0.3 / 0.7,0.5 / 0.5$, and $0.7 / 0.3$. These ratios correspond

\footnotetext{
${ }^{1}$ Ghent University / IBBT, Dept. of Information Technology, Gaston Crommenlaan 8 box 201, B-9050 Ghent, Belgium, e-mail: emmeric.tanghe@intec.ugent.be.

${ }^{2}$ University of Lille, IEMN, Group TELICE, Bldg. P3, F 59655 Villeneuve d'Ascq, France, e-mail: davy.gaillot@univ-lille1.fr.
} 
to common distributions of power between the DMC and SC parts reported in literature [4].

Following, the SC parameter vector estimates $\widehat{\boldsymbol{\theta}}_{\boldsymbol{S C}}$ were calculated with unitary ESPRIT, SAGE, and RiMAX for the 1000 constructed channels. The pairing of each estimated SC with its exact ray-traced counterpart is done in terms of smallest Multipath Component Distance (MCD) between both [5]

\section{RESULTS}

Fig. 1 shows the Complementary Cumulative Distribution Functions (CCDFs) of the absolute errors between AOAs of estimated and ray-traced SCs. CCDFs are shown for each of the three estimation algorithms and each of the three $P_{D M C} / P_{S C}$ ratios. As expected, the DMC-inclusive RiMAX algorithm exhibits better error performance (CCDFs shifted to the left) than the ESPRIT and SAGE algorithms. We note that SAGE returns slightly better angular estimates than ESPRIT.

Furthermore, for all three algorithms the $P_{D M C} / P_{S C}$ scenarios do not appear to have a large impact on the $\mathrm{SC}$ estimator performance. As expected, the effect of the $P_{D M C} / P_{S C}$ ratio on RiMAX performance is almost nonexistent as this algorithm correctly accounts for DMC. For ESPRIT and SAGE, larger relative DMC power does not necessarily mean worse SC estimates, showing that even at the largest $P_{D M C} / P_{S C}$ ratio, the DMC power is not high enough to overshadow the strongest SCs in this simulation setup.

Table 1 shows SC parameter values corresponding to worst-case exceedance probabilities (i.e., the probability that an error occurs that is larger than that value) of 50,10 , and $1 \%$. Three values are shown per parameter and exceedance probability, corresponding to estimations with the ESPRIT, SAGE, and RiMAX algorithms respectively. The values in Table 1 are averaged values taken over all three $P_{D M C} / P_{S C}$ scenarios. From Table 1, it is clear that even at the larger, more forgiving exceedance probabilities of $50 \%$ and $10 \%$, ESPRIT and SAGE show large errors compared to the relatively small errors exhibited by RiMAX.

\section{CONCLUSIONS}

The results of this study demonstrate that specular multipath component estimation in the presence of dense multipath components is prone to large estimation errors if the signal model is not accordingly modified in estimation algorithms such as ESPRIT and SAGE. Therefore, determining the DMC by subtracting the specular part, estimated by ESPRIT or SAGE, from the total channel response, as it is frequently done in literature, is flawed and must be avoided.

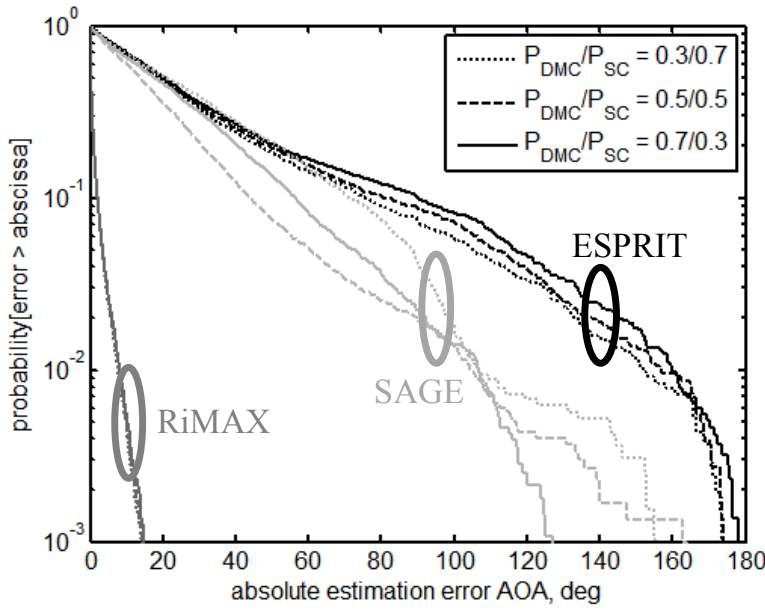

Figure 1: CCDFs of absolute AoA estimation error.

\begin{tabular}{|c|l|l|l|}
\hline Exceedance & \multicolumn{1}{|c|}{$50 \%$} & \multicolumn{1}{|c|}{$10 \%$} & \multicolumn{1}{|c|}{$1 \%$} \\
\hline \multirow{3}{*}{ AOA [deg] } & (E) 19.7 & (E) 82.2 & (E) 156.0 \\
& (S) 17.4 & (S) 57.2 & (S) 106.0 \\
& (R) 0.3 & (R) 2.1 & (R) 7.9 \\
\hline \multirow{3}{*}{ AOD [deg] } & (E) 21.9 & (E) 108.9 & (E) 171.3 \\
& (S) 15.6 & (S) 71.8 & (S) 128.3 \\
& (R) 0.2 & (R) 1.6 & (R) 6.3 \\
\hline \multirow{3}{*}{ TOA [ns] } & (E) 7.7 & (E) 25.3 & (E) 35.0 \\
& (S) 10.5 & (S) 27.0 & (S) 35.3 \\
& (R) 0.3 & (R) 2.4 & (R) 7.1 \\
\hline \multirow{3}{*}{ Power [dB] } & (E) 41.6 & (E) 60.4 & (E) 76.4 \\
& (S) 9.8 & (S) 35.8 & (S) 59.4 \\
& (R) 0.2 & (R) 2.7 & (R) 9.3 \\
\hline
\end{tabular}

Table 1: average errors corresponding to exceedances of 50,10 , and $1 \%$; $(\mathrm{E})=\mathrm{ESPRIT},(\mathrm{S})=\mathrm{SAGE}$, and (R) $=$ RiMAX

\section{References}

[1] M. Haardt, "Efficient One-, Two-, and Multidimensional High-Resolution Array Signal Processing," PhD thesis, 1996.

[2] B. H. Fleury, M. Tschudin, R. Heddergott et al., "Channel Parameter Estimation in Mobile Radio Environments Using the SAGE Algorithm," IEEE Journal on Selected Areas in Communications, 17(3):434-450, March 1999.

[3] A. Richter, "Estimation of Radio Channel Parameters: Models and Algorithms," PhD thesis, 2005.

[4] J. Salmi, J. Poutanen, K. Haneda et al., "Incorporating Diffuse Scattering in Geometrybased Stochastic MIMO Channel Models," 4th European Conference on Antennas and Propagation, Barcelona, ES, April 2010.

[5] M. Steinbauer, H. Özcelik, H. Hofstetter et al., "How to Quantify Multipath Separation," IEICE Transactions on Electronics, E85-C(3):552557, 2002. 